\title{
Determinant Versus Permanent
}

\author{
Manindra Agrawal
}

\begin{abstract}
We study the problem of expressing permanent of matrices as determinant of (possibly larger) matrices. This problem has close connections with complexity of arithmetic computations: complexities of computing permanent and determinant roughly correspond to arithmetic versions of the classes NP and P respectively. We survey known results about their relative complexity and describe two recently developed approaches that might lead to a proof of the conjecture that permanent can only be expressed as determinant of exponential-sized matrices.
\end{abstract}

Mathematics Subject Classification (2000). Primary 68Q17; Secondary 68W30.

Keywords. Arithmetic computation, complexity classes, determinant, permanent.

\section{Introduction}

Determinant of square matrices plays a fundamental role in linear algebra. It is a linear function on rows (and columns) of the matrix, and has several nice interpretations. Geometrically, it is the volume of the parallelopied defined by rows (or columns) of the matrix, and algebraically, it is the product of all eigenvalues, with multiplicity, of the matrix. It also satisfies a number of other interesting properties, e.g., it is multiplicative, invariant under linear combinations of rows (and columns) etc. Permanent of a square matrix is a number that is defined in a way similar to the determinant. For matrix $X=\left[x_{i, j}\right]_{1 \leq i, j \leq n}$,

$$
\text { per } X=\sum_{\pi \in S_{n}} \prod_{i=1}^{n} x_{i, \pi(i)} \text {, }
$$

where $S_{n}$ is the symmetric group on $n$ elements. The only difference with determinant is in the signs of terms:

$$
\operatorname{det} X=\sum_{\pi \in S_{n}} \operatorname{sgn}(\pi) \cdot \prod_{i=1}^{n} x_{i, \pi(i)},
$$

where $\operatorname{sgn}(\pi) \in\{1,-1\}$ is the sign of permutation $\pi{ }^{1}$ Despite the similarity in definition, permanent has much fewer properties than determinant. No nice

\footnotetext{
${ }^{1}$ Both permanent and determinant are special forms of immanents defined as $\operatorname{imm}_{\chi} X=$ $\sum_{\pi \in S_{n}} \chi(\pi) \cdot \prod_{i=1}^{n} x_{i, \pi(i)}$ where $\chi: S_{n} \mapsto \mathbb{C}$ is a character of $S_{n}$. For permanent, $\chi=i d$ and for determinant, $\chi$ equals sign of permutation.
} 
geometric or algebraic interpretation is known for permanent; and it is neither multiplicative nor invariant under linear combinations of rows or columns. Perhaps for this reason, permanent did not get much attention until late 1970s, and just about everything known about it until then is in the book [10]. In 1979, Leslie Valiant [24] completely changed the view on permanent by showing that complexity of computing permanent precisely captures the arithmetic version of class NP, called VNP. Since then, properties of permanent have been extensively studied by complexity theorists.

One of the most natural question about permanent is to investigate its relationship with determinant. It is easy to see that determinant of matrix $X$ can be expressed as permanent of a related matrix $\hat{X}$ whose entries are 0,1 , or $x_{i, j} \mathrm{~s}$ and size is $O(n)$ (set up entries of $\hat{X}$ such that $\operatorname{det} \hat{X}=\operatorname{det} X$ and the product corresponding to every permutation that has an even cycle is zero). For the converse, the best known bound on the size of matrix $\hat{X}$ whose entries are constants and $x_{i, j} \mathrm{~s}$, and $\operatorname{det} \hat{X}=\operatorname{per} X$ is $2^{\Omega(n)}$. This suggests that complexity of computing permanent is much higher than that of determinant. Although widely believed, this remains a conjecture. This conjecture has a close connection to the conjectured separation of arithmetic NP from arithmetic P (the class of all functions that can be efficiently computed by arithmetic operations, see next section for a precise definition). It is known that complexity of determinant is close to the complexity of arithmetic P: every function computed by $n$ arithmetic operations can be expressed as determinant of a matrix of size $n^{O(\log n)}$. This lends more importance to the problem of settling the conjecture.

There have been some attempts to answer the conjecture positively $[14,6,15,8]$. A sequence of arithmetic operations can be modeled as an arithmetic circuit, and the size of arithmetic circuit denotes the number of arithmetic operations in the sequence. In [8], monotone circuits were considered, these are circuits in which no constant is negative. For computability by such restricted circuits, an exponential lower bound was shown for the complexity of permanent. A different restriction on arithmetic circuits is that of depth - the number of layers of operations. These circuits were considered in $[14,19,6]$ and lower bounds were shown on the complexity of computing permanent by depth three circuits. Finally, [15] considers yet another restriction. In this restriction, every gate of the circuit is required to compute a multi-linear polynomial. A superpolynomial lower bound is shown on formulas (circuits with outdegree one) of this kind computing permanent.

All the above lower bounds hold for very restricted settings, and the techniques used do not seem to generalize. Over the last few years, however, two new techniques have been proposed that hold some promise. The first of these was proposed by Mulmuley and Sohoni [11]. They transform the problem to algebraic geometry domain where it is reduced to showing that the permanent polynomial does not lie in the closure of a certain orbit of determinant polynomial.

The second approach was proposed by Kabanets and Impagliazzo [9]. They reduced the problem to that of finding a deterministic, subexponential-time algorithm for the Identity Testing. Identity Testing problem is defined as follows: given an arithmetic circuit computing polynomial $p$ over $n$ variables, test if $p=0$. There 
exist several randomized polynomial-time algorithms for solving this. Kabanets and Impagliazzo show that any deterministic, subexponential-time algorithm for the problem will imply either a superpolynomial lower bound for arithmetic circuits computing permanent, or a boolean lower bound on the class NEXP. This connection was strengthened in [1] to show that if there exist special kinds of deterministic algorithms for testing identities given by superconstant depth arithmetic circuits, then permanent requires superpolynomial sized arithmetic circuits.

In this article, we will describe the known results on lower bounds on permanent as well as the two new approaches outlined above.

\section{Definitions}

$\mathbb{Q}, \mathbb{R}$, and $\mathbb{C}$ are respectively fields of rational numbers, real numbers, and complex numbers.

An arithmetic circuit over field $F$ is a directed, acyclic graph with labelled vertices. Vertices of indegree zero are labelled with either a variable $x_{i}$ or a constant from $F$. Vertices labelled with variables are called input gates. The remaining vertices are labelled with either ' + ' or '*' ${ }^{*}$ and are called addition or multiplication gates respectively. Vertices with outdegree zero are also called output gates. We restrict our attention to circuits with exactly one output gate. The fanin of a gate equals the number of edges incident to the gate. In this article, gates have unbounded fanin when not mentioned otherwise. The size of circuit $C$ equals the number of gates in it. The depth of circuit $C$ equals the length of the longest path from an input gate to output gate. The degree of $C$ is inductively defined as: the degree of an input gate is one or zero depending on whether it is labelled by a variable or constant; degree of an addition gate is the maximum of the degree of the gate whose edges are incident to the gate; degree of a multiplication gate is the sum of the degrees of the gate whose edges are incident to the gate; finally, the degree of $C$ is the degree of its output gate.

An arithmetic circuit $C$ computes a polynomial as follows. The polynomial computed at an input gate equals the label of the gate. For any other gate $g$, let $g_{1}, \ldots, g_{k}$ be all the gates that have an edge incident to $g$ and let $p_{g_{i}}$ be the polynomial computed at gate $g_{i}$. Then the polynomial computed at the gate $g$ equals $\sum_{i=1}^{k} p_{g_{i}}$ if $g$ is an addition gate, and equals $\prod_{i=1}^{k} p_{g_{i}}$ if $g$ is a multiplication gate. The polynomial computed by the circuit is the polynomial computed at its output gate.

Let $\left\{p_{n}\right\}_{n>0}$ be a family of polynomials with $p_{n}$ a polynomial of degree $d(n)$ over $n$ variables. A circuit family $\left\{C_{n}\right\}_{n>0}$ is said to compute $\left\{p_{n}\right\}$ if for every $n$, the polynomial computed by $C_{n}$ equals $p_{n}$. In the following, we will write a family $\left\{p_{n}\right\}_{n>0}$ simply as $\left\{p_{n}\right\}$.

Arithmetic branching programs are a restricted form of arithmetic circuits in which every multiplication gate has fanin exactly two, and in addition, at least one of the two gates, from which edges are incident to the multiplication gate, is an input gate. Such circuits are also called skew circuits. 
The class $\mathrm{VP}_{F}$, the arithmetic analog of class $\mathrm{P}$, is defined to be the set of polynomial families $\left\{p_{n}\right\}$ over field $F$ such that (1) each $p_{n}$ is of degree $n^{O(1)}$, (2) there exists a circuit family $\left\{C_{n}\right\}$ computing $\left\{p_{n}\right\}$ such that $C_{n}$ is of size $n^{O(1)} .^{2}$ The class $\mathrm{VNP}_{F}$, the arithmetic analog of class $\mathrm{NP}$, is defined to be the set of polynomial families $\left\{p_{n}\right\}$ over field $F$ such that (1) each $p_{n}$ is of degree $n^{O(1)}$, (2) there exists a family of polynomials $\left\{q_{n}\right\} \in \mathrm{VP}_{F}$ such that for every $n$,

$$
p_{n}\left(x_{1}, x_{2}, \ldots, x_{n}\right)=\sum_{y_{1}=0}^{1} \sum_{y_{2}=0}^{1} \cdots \sum_{y_{m}=0}^{1} q_{n+m}\left(x_{1}, x_{2}, \ldots, x_{n}, y_{1}, y_{2}, \ldots, y_{m}\right)
$$

with $m=n^{O(1)} .^{3}$

Given two polynomials $p\left(x_{1}, x_{2}, \ldots, x_{n}\right)$ and $q\left(y_{1}, y_{2}, \cdots, y_{m}\right)$ over field $F$, we say that $p$ is a projection of $q$ if $p\left(x_{1}, x_{2}, \ldots, x_{n}\right)=q\left(\alpha_{1}, \alpha_{2}, \cdots, \alpha_{m}\right)$ where each $\alpha_{i} \in F \cup\left\{x_{1}, x_{2}, \ldots, x_{n}\right\}$. Given two polynomial families $\left\{p_{n}\right\}$ and $\left\{q_{n}\right\}$, we say that $\left\{p_{n}\right\}$ is a projection of $\left\{q_{n}\right\}$ if for every $n$ there exists an $m=n^{O(1)}$ such that $p_{n}$ is a projection of $q_{m} \cdot{ }^{4}$

Let $\operatorname{per}_{F}=\left\{\operatorname{per}_{F, n}\right\}$ and $\operatorname{det}_{F}=\left\{\operatorname{det}_{F, n}\right\}$ denote the families of permanent and determinant polynomials over field $F$ respectively. Both these families have polynomials over $n^{2}$ variables for each $n$.

Valiant [24] proved that:

Theorem 2.1 ([24]). For any $F$, per $_{F} \in \mathrm{VNP}_{F}$. In addition, for any $F, \operatorname{char}(F) \neq$ 2 , for any polynomial family $\left\{p_{n}\right\}$ in $\mathrm{VNP}_{F},\left\{p_{n}\right\}$ is a p-projection of $\operatorname{per}_{F}$.

So permanent is as hard to compute as any polynomial family in VNP. In contrast, determinant can be computed efficiently. A nice characterization of determinant was shown in $[4,21,25]$ :

Theorem 2.2 ([4, 21, 25]). For any $F$, $\operatorname{det}_{F}$ can be computed by polynomial-sized arithmetic branching programs. In addition, for any $F$ and for any polynomial family $\left\{p_{n}\right\}$ computed by polynomial-sized arithmetic branching programs, $\left\{p_{n}\right\}$ is a p-projection of $\operatorname{det}_{F}$.

In fact, all families in VP are almost p-projections of determinant.

Theorem 2.3 ([23]). Let $C$ be a circuit of size s computing a polynomial of degree $d$. There exists another circuit computing the same polynomial of size $s^{O(1)}$ and depth $O(\log s+\log d)$.

Corollary 2.4. Any circuit family in $\mathrm{VP}_{F}$ can be computed by circuit families of polynomial size and logarithmic depth.

Corollary 2.5. For every circuit family $\left\{p_{n}\right\} \in \mathrm{VP}_{F}$ and for every $n, p_{n}$ is a projection of $\operatorname{det}_{F, m}$ where $m=n^{O(\log n)}$.

\footnotetext{
${ }^{2}$ In addition, circuit $C_{n}$ must be efficiently computable given $1^{n}$. This property does not seem to play any role in obtaining lower bounds.

${ }^{3}$ The class $\# \mathrm{P}_{F}$ is the 'functional' version of class $\mathrm{VNP}_{F}$ : a polynomial family $\left\{p_{n}\right\} \in \mathrm{VNP}_{F}$ belongs to $\# \mathrm{P}_{F}$ when for each $n, p_{n}$ is viewed as a map from $F^{n}$ to $F$.

${ }^{4}$ Again, given $1^{n}$, the projection specified by $\left(\alpha_{1}, \alpha_{2}, \ldots, \alpha_{m}\right)$ should be efficiently computable.
} 
The above characterizations of complexities of determinant and permanent imply that, in order to separate $\mathrm{VP}_{F}$ from $\mathrm{VNP}_{F}$, it is enough to show that $\operatorname{per}_{F}$ is not an almost p-projection of $\operatorname{det}_{F}$ (in the sense above).

\section{Known Lower Bounds on Permanent}

Lower bounds are known on permanent only for restricted circuits. In this section, we describe important lower bounds of this kind. Three major restrictions have been considered for proving such lower bounds: monotone circuits, constant depth circuits, and multilinear formulas.

3.1. Monotone Circuits. A circuit over $\mathbb{Q}$ or $\mathbb{R}$ is monotone if all the constants in the circuit are non-negative. This is a very restricted class of circuits since no cancellations can occur in it. Jerrum and Snir [8] showed that any monotone circuit family that computes permanent must have exponential size.

3.2. Constant Depth Circuits. In this restriction, the depth of a circuit family is fixed, i.e., it is independent of $n$. Permanent (or any polynomial of degree $n^{O(1)}$ for that matter) can be computed by an exponential size depth two circuit family. Conversely, it is easy to see that any depth two circuit family computing permanent must have exponential size.

Depth three circuit families are, however, non-trivial. A depth three circuit can be of the form "sum-of-products-of-sums" or "product-of-sums-of-products." The latter form can easily be seen to require exponential size to compute permanent (the topmost multiplication gate can be shown to be redundant transforming the circuit to a depth two circuit). Circuit families of the first form are powerful: Ben Or observed that they can efficiently compute all symmetric polynomials of degree $n^{O(1)}$ over fields of characteristic zero.

The best known lower bound in fields of characteristic zero is by Shpilka and Wigderson [19] who prove that permanent (and determinant) requires a circuit family of size $\Omega\left(n^{2}\right)$. Their idea is to consider the space spanned by all partial derivatives of the polynomials computed at each gate of a given circuit. They show that for a depth three circuit with small size, the space spanned by the derivatives of output polynomial would be of small dimension while the space spanned by derivatives of permanent is of large dimension.

Over finite fields, the situation is better. Grigoriev and Razborov [6] showed an exponential lower bound on the size of depth three circuit families computing determinant and permanent. Their approach was to show that polynomial computed by a depth three circuit of small size can be 'approximated' by a low-degree polynomial (approximated in the sense that the two polynomials agree on large set of points from the field). Then they observed that determinant and permanent cannot be approximated by low-degree polynomials. 
3.3. Multilinear Formulas. Multilinear formulas are circuits such that (1) the outdegree of every gate is at most one, and (2) the polynomial computed at every gate is multilinear. Such circuits have severely limited multiplication gates - the polynomials input to a multiplication gate must be over disjoint sets of variables. Using a combination of partial derivative technique and random restrictions (setting some input variables to random values), Raz [15] proved a lower bound of $n^{\Omega(\log n)}$ on the size of families of multilinear formulas computing permanent and determinant.

\section{The Algebraic Geometry Approach}

Mulmuley and Sohoni [11] have offered a completely new approach to the problem of proving a lower bound on permanent for unrestricted circuits by transforming the problem to geometric settings. In this section, we give a brief overview of their approach.

Suppose, for $F=\mathbb{Q}, \operatorname{per}_{F, n}$ is a projection of $\operatorname{det}_{F, m}, m>n$. Define $\widehat{\operatorname{per}}_{F, m}=$ $x_{m^{2}}^{m-n} \cdot \operatorname{per}_{F, n}$. It follows that $\widehat{\operatorname{per}}_{F, m}$ is also a projection of $\operatorname{det}_{F, m}$ (just multiply all constants of the projection by $x_{m^{2}}$ ). This can be written as

$$
\widehat{\operatorname{per}}_{F, m}\left(x_{1}, x_{2}, \ldots, x_{m^{2}}\right)=A \cdot \operatorname{det}_{F, m}=\operatorname{det}_{F, m}\left(\left(x_{1}, x_{2}, \ldots, x_{m^{2}}\right) \cdot A\right),
$$

where $A$ is a $m^{2} \times m^{2}$ matrix over $\mathbb{Q}$. Matrix $A$ would be singular whenever $m>n$ since the variables $x_{n^{2}+1}, \ldots, x_{m^{2}-1}$ do not occur in $\widehat{\operatorname{per}}_{F, m}$. Let $A_{\bar{\epsilon}}$ be a slight 'perturbation' of $A$ obtained by adding $\epsilon_{i, j}$ to the $(i, j)$ th entry of $A$. For nearly all values of $\bar{\epsilon}$ close to zero, $A_{\bar{\epsilon}}$ is non-singular and the polynomial $A_{\bar{\epsilon}} \cdot \operatorname{det}_{F, m}$ approximates the polynomial $\widehat{\operatorname{per}}_{F, m}$ very well (all the coefficients of two polynomials are close to each other). Now consider the space $V=\mathbb{C}^{M}$ with $M=\left(\begin{array}{c}m^{2}+m-1 \\ m\end{array}\right)$. Every homogeneous polynomial of degree $m$ over $m^{2}$ variables can be viewed as a point in this space (degree $m$ monomials forming the basis). So both $\operatorname{det}_{F, m}$ and $\widehat{\text { per }}_{F, m}$ are points in $V$ (since $F=\mathbb{Q}$ and both polynomials are of degree $m$ over $m^{2}$ variables). Let $O$ be the orbit of $\operatorname{det}_{F, m}$ under the action of $\mathrm{GL}_{m^{2}}(\mathbb{C})$, i.e.,

$$
O=\left\{B \cdot \operatorname{det}_{F, m} \mid B \text { is an invertible matrix over } \mathbb{C}\right\} .
$$

Set $O$ can be viewed as a set of points in $V$. The above argument shows the following:

Lemma 4.1 ([11]). If $\operatorname{per}_{F, n}$ is a projection of $\operatorname{det}_{F, m}$ then the point corresponding to $\widehat{\mathrm{per}}_{F, m}$ in $V$ lies in the closure of the set $O$ in $V$. Conversely, if $\widehat{\mathrm{per}}_{F, m}$ lies in the closure of $O$ then $\operatorname{per}_{F, n}$ can be approximated by projections of $\operatorname{det}_{F, m}$ to any desired accuracy.

This (near) characterization is the staring point of their approach. Instead of $V$, we can work in the projective space $P(V)$ too since both the polynomials are homogeneous. The same near characterization holds in $P(V)$ as well with 
$\mathrm{GL}_{m^{2}}(\mathbb{C})$ replaced by $\mathrm{SL}_{m^{2}}(\mathbb{C})$, the group of all matrices with determinant one. The advantage of working in $P(V)$ is that the closure of $O$ (under the classical Euclidean topology) coincides with the closure of $O$ under Zariski topology [12]. In Zariski topology, there is the well-studied notion of stability that captures this problem: $\operatorname{det}_{F, m}$ is $\widehat{\operatorname{per}}_{F, m}$-stable under $\mathrm{SL}_{m^{2}}(\mathbb{C})$ if $\widehat{\operatorname{per}}_{F, m}$ lies in the closure of the orbit $O$ (we abuse the notation here by using the same names as in $V$ for polynomials and sets in $P(V))$.

Points in the orbit $O$ have a useful property. For any point $p \in P(V)$, let

$$
G_{p}=\left\{A \in \mathrm{SL}_{m^{2}}(\mathbb{C}) \mid A \cdot p=p\right\} .
$$

Group $G_{p}$ is called the stabilizer of $p$.

Lemma 4.2. For any point $p \in O, G_{p}$ is a conjugate of $G_{\operatorname{det}_{F, m}}$.

Proof. Let $p=B \cdot \operatorname{det}_{F, m} \in O$. Then $G_{p}=B \cdot G_{\operatorname{det}_{F, m}} \cdot B^{-1}$.

Suppose the orbit of polynomial $\widehat{\mathrm{per}}_{F, m}$ under $\mathrm{SL}_{m^{2}}(\mathbb{C})$ is a closed set (such polynomial are called stable). Let $Q$ be the orbit of $\widehat{\operatorname{per}}_{F, m}$ under $\mathrm{SL}_{m^{2}}(\mathbb{C})$. By Luna's slice theorem, there is a neighborhood $N$ of $Q$ such that for any point $p \in N, G_{p}$ is a conjugate of a subgroup of $G_{\widehat{\mathrm{per}}_{F, m}}$. Since closure of $O$ contains $\widehat{\operatorname{per}}_{F, m}$, there is a point in $N$, say $q$, such that $q=B \cdot \operatorname{det}_{F, m}$. This means $G_{q}$ is a conjugate of $G_{\operatorname{det}_{F, m}}$. Therefore, $G_{\operatorname{det}_{F, m}}$ is a conjugate of a subgroup of $G_{\widehat{\operatorname{per}}_{F, m}}$. On the other hand, it is well known that $G_{\operatorname{det}_{F, m}}$ is 'larger' than $G_{\widehat{\operatorname{per}}_{F, m}}$ : $G_{\operatorname{det}_{F, m}}$ is characterized by the transformations of the kind $X \mapsto A \cdot X \cdot B^{-1}$ where $A, B \in \mathrm{GL}_{m}(\mathbb{C})$ while $G_{\widehat{\operatorname{per}}_{F, m}}$ is characterized by the transformations of the kind $X \mapsto A \cdot X \cdot B^{-1}$ where $A, B \in \mathrm{GL}_{m}(\mathbb{C})$ and both $A$ and $B$ are either diagonal or permutation matrices. Therefore, $G_{\operatorname{det}_{F, m}}$ cannot be a conjugate of a subgroup of $G_{\widehat{p e r}_{F, m}}$. (This is a rough argument; to make it precise, more work is needed.)

Unfortunately, $\widehat{\operatorname{per}}_{F, m}$ is not stable (interestingly, $\operatorname{per}_{F, n}$ is stable in the smaller dimensional space defined by degree $n$ homogeneous polynomials over $n^{2}$ variables; the translation to higher dimensional space ruins the stability). Mulmuley and Sohoni define the notion of partial stability and show that $\widehat{\operatorname{per}}_{F, m}$ is partially stable. Now their aim is to make the above argument work even for partially stable points. A more detailed explanation of their approach is in [16].

\section{The Derandomization Approach}

Kabanets and Impagliazzo [9] have discovered another new approach for proving lower bounds on permanent. Unlike the previous one, this approach is based on arithmetic circuits. In this section, we outline their approach and its variation in $[1]$.

The Identity Testing problem is defined as follows: given an arithmetic circuit $C$ over field $F$ as input, decide if the polynomial computed by the circuit is the zero polynomial. This is a classical problem in computational algebra and there exist 
several randomized polynomial-time algorithms for it. Perhaps the simplest one is by Schwartz and Zippel [17, 26]: randomly choose values for variables of $C$ from a set in $F$ of size $2 d$, here $d$ is the degree of $C$ (if $|F|<2 d$ then extend $F$ slightly); output ZERO if $C$ evaluates to zero, otherwise NON-ZERO. An easy argument shows that this test is correct with probability at least $\frac{1}{2}$ when $C$ computes a non-zero polynomial and always correct when $C$ computes a zero polynomial.

Kabanets and Impagliazzo show that if there exists a deterministic subexponential $\left(=2^{n^{o(1)}}\right)$ time algorithm for solving Identity Testing problem then at least one of the following two lower bounds hold:

1. NEXP requires superpolynomial sized boolean circuits.

2. Permanent requires superpolynomial sized arithmetic circuits.

To see this, suppose that permanent has polynomial sized arithmetic circuits for some field $F$ of characteristic different from two. Consider a non-deterministic machine that, on input $1^{n}$, guesses the circuit that computes per $_{F, n}$ and verifies it to be correct. It does this by inductively verifying that the circuit, under appropriate settings of its inputs, computes per $_{F, n-1}$ correctly and then verifying the equation for $\operatorname{per}_{F, n}$ that expresses it in terms of per $_{F, n-1}$. Verifying the equation is an instance of Identity Testing problem and so can be done in subexponential time by assumption. Therefore, given any matrix $A \in F^{n^{2}}$, per $A$ can be computed in non-deterministic subexponential time. Now assume that NEXP has polynomial sized boolean circuits. By [3, 22], it follows that $\mathrm{NEXP} \subseteq \mathrm{P}^{\# \mathrm{P}}$. Since complexity of \#P is exactly the complexity of computing permanent, it follows that NEXP is in non-deterministic subexponential time contradicting the non-deterministic time hierarchy theorem [18].

This result falls short of pointing a way for proving lower bounds on permanentbesides finding a deterministic algorithm for Identity Testing, one needs to assume NEXP has polynomial sized boolean circuits which is very unlikely to be true. However, it does point to a connection between Identity Testing problem and permanent lower bounds. This connection was strengthened in [1] by defining pseudo-random generators for arithmetic circuits. Pseudo-random generators in the boolean settings have been studied intensively (see, e.g., [5, 13, 7, 20]). It is known that constructing pseudo-random generators is equivalent to proving lower bounds in the boolean settings. In [1], pseudo-random generators are defined in arithmetic settings and a similar equivalence is observed.

Let $\mathcal{A C}_{F}$ be the class of all arithmetic circuits over $F$ and $\mathcal{A}_{F} \subseteq \mathcal{A C}_{F}$.

Definition 5.1. Function $f: \mathbb{N} \mapsto(F[y])^{*}$ is a $(\ell(n), n)$-pseudo-random generator against $\mathcal{A}_{F}$ if:

- $f(n) \in(F[y])^{n+1}$ for every $n>0$.

- Let $f(n)=\left(f_{1}(y), \ldots, f_{n}(y), g(y)\right)$. Then each $f_{i}(y)$ as well as $g(y)$ is a polynomial of degree at most $2^{\ell(n)}$.

- For any circuit $C \in \mathcal{A}_{F}$ of size $n$ with $m \leq n$ inputs:

$$
C\left(x_{1}, x_{2}, \ldots, x_{m}\right)=0 \text { iff } C\left(f_{1}(y), f_{2}(y), \ldots, f_{m}(y)\right)=0(\bmod g(y)) .
$$


A direct application of Schwartz-Zippel lemma [17, 26] shows that there always exist $(O(\log n), n)$-pseudo-random generators against $\mathcal{A C}_{F}$. Call such generators optimal pseudo-random generators. Pseudo-random generators that can be efficiently computed are of special interest.

Definition 5.2. A $(\ell(n), n)$-pseudo-random generator $f$ against $\mathcal{A}_{F}$ is efficiently computable if $f(n)$ is computable in time $2^{O(\ell(n))}$.

An easy argument shows that if there exists an efficiently computable $(\ell(n), n)$ pseudo-random generator against $\mathcal{A C}_{F}$ then the Identity Testing problem can be solved deterministically in time $2^{O(\ell(n))}$ : evaluate the given circuit $C$ of size $n$ modulo $g(y)$ after substituting for $i^{t h}$ input variable polynomial $f_{i}(y)$ where $f(n)=\left(f_{1}(y), \ldots, f_{n}(y), g(y)\right)$. In particular, if there exist an efficiently computable optimal pseudo-random generator against $\mathcal{A C}_{F}$ then Identity Testing can be solved in polynomial time.

An efficiently computable pseudo-random generator also results in a lower bound.

Theorem $5.3([1])$. Let $f$ be an efficiently computable $(\ell(n), n)$-pseudo-random generator against $\mathcal{A}_{F}$. Then there is a multilinear polynomial over $2 \ell(n)$ variables, computable in time $2^{O(\ell(n))}$, that cannot be computed by any circuit in $\mathcal{A}_{F}$ of size $n$.

Proof. For any $m=\ell(n)$, define polynomial $q_{f}\left(x_{1}, x_{2}, \ldots, x_{2 m}\right)$ as:

$$
q_{f}\left(x_{1}, x_{2}, \ldots, x_{2 m}\right)=\sum_{S \subseteq[1,2 m]} c_{S} \cdot \prod_{i \in S} x_{i} .
$$

The coefficients $c_{S}$ satisfy the condition

$$
\sum_{S \subseteq[1,2 m]} c_{S} \cdot \prod_{i \in S} f_{i}(y)=0
$$

where $f(n)=\left(f_{1}(y), f_{2}(y), \ldots, f_{n}(y), g(y)\right)$. Such a $q_{f}$ always exists as the following argument shows.

The number of coefficients of $q_{f}$ are exactly $2^{2 m}$. These need to satisfy a polynomial equation of degree at most $2 m \cdot 2^{m}$. So the equation gives rise to at most $2 m \cdot 2^{m}+1$ homogeneous constraints on the coefficients. Since $\left(2 m \cdot 2^{m}+1\right)<2^{2 m}$ for $m \geq 3$, there is always a non-trivial polynomial $q_{f}$ satisfying all the conditions.

The polynomial $q_{f}$ can be computed by solving a system of $2^{O(m)}$ linear equations in $2^{O(m)}$ variables over the field $F$. Each of these equations can be computed in time $2^{O(m)}$ using computability of $f$. Therefore, $q_{f}$ can be computed in time $2^{O(m)}$. Now suppose $q_{f}$ can be computed by a circuit $C \in \mathcal{A}_{F}$ of size $n$. By the definition of polynomial $q_{f}$, it follows that $C\left(f_{1}(y), f_{2}(y), \ldots, f_{2 m}(y)\right)=0$. The size of circuit $C$ is $n$ and it computes a non-zero polynomial. This contradicts the pseudo-randomness of $f$. 
A partial converse of this theorem can also be shown: if there exists a polynomial family computable in time $2^{O(\ell(n))}$ that cannot be computed by any size $n$ circuit family in $\mathcal{A}_{F}$ then there exists an efficiently computable $\left(\ell^{2}(n), n\right)$-pseudorandom generator against $\mathcal{A}_{F}$, when the degree of every size $n$ circuit in $\mathcal{A}_{F}$ is bounded by $n^{O(1)}$.

An efficient optimal pseudo-random generator against $\mathcal{A C}_{F}$ yields a polynomial that requires exponential (in the number of variables) sized circuits. However, it is not clear whether the polynomial $q_{f}$ can be computed as permanent of a matrix of size $m^{O(1)}$. To get this, one needs to show that all the coefficients $c_{S}$ of $q_{f}$ are themselves efficiently computable. If this is done, then using the VNP characterization of permanent, it follows that $q_{f}$ equals permanent of a matrix of size $m^{O(1)}$. This results in an exponential lower bound on permanent.

For a superpolynomial lower bound, one needs either an $\left(n^{o(1)}, n\right)$-pseudo random generator against $\mathcal{A C}_{F}$ or an optimal pseudo-random generators against a much smaller class of circuits.

Theorem 5.4 ([1]). Let $f$ be an efficiently computable optimal pseudo-random generator against the class of circuits of depth $\omega(1)$ such that the associated polynomial $q_{f}$ is in VNP. Then permanent cannot computed by any polynomial sized circuit.

Proof. From the previous theorem, it follows that the polynomial $q_{f}$ cannot be computed by exponential sized circuits of depth $\omega(1)$. A size $n^{d}$, depth $d \log n$ arithmetic circuit with fanin two multiplication gates can be translated to a subexponential sized depth $d$ circuit by "cutting" the circuit into $\log n$ layers of depth $d$ each, and then "flattening" each layer to a subexponential sized circuit of depth two. Since every polynomial sized circuit computing permanent can be transformed to a depth $O(\log n)$, size $n^{O(1)}$ circuit with fanin two multiplication gates [23], the theorem follows.

It is not clear at the moment how to construct optimal pseudo-random generators against constant depth circuits. In [1] a generator is conjectured. Unconditionally, we only know generators against depth two, polynomial sized circuits (the proof is easy, see [1]). We know an optimal generator against the following very special class of circuits too:

$$
\mathcal{A}=\left\{C_{n}(x) \mid C_{n}(x)=(1+x)^{n}-1-x^{n} \text { over ring } Z_{n}\right\}
$$

Notice that the circuits in the class $\mathcal{A}$ are not over a fixed field (or ring), and the size of the circuit $C_{n}$ is $O(\log n)$ and the degree is $n$. In [2], the following optimal generator was constructed against $\mathcal{A}$ :

$$
f(m)=\left(x, 0, \ldots, 0, x^{16 m^{5}} \cdot \prod_{r=1}^{16 m^{5}} \prod_{a=1}^{4 m^{4}}\left((x-a)^{r}-1\right)\right)
$$




\section{Concluding Remarks}

The problem of proving that permanent of size $n$ matrix cannot be expressed as determinant of size $n^{O(\log n)}$ matrix is of great importance in complexity theory. While the existing approaches have failed to shed light on this, one hopes that at least one of the two new approaches will eventually lead to a solution of the problem.

\section{Acknowledgements}

I wish to thank Somenath Biswas for enjoyable discussions and help in preparing this article.

\section{References}

[1] M. Agrawal. Proving lower bounds via pesudo-random generators. In Proceedings of the FSTESTCS, pages 96-105, 2005.

[2] Manindra Agrawal. On derandomizing tests for certain polynomial identities. In Proceedings of the Conference on Computational Complexity, pages 355-362, 2003.

[3] L. Babai, L. Fortnow, N. Nisan, and A. Wigderson. BPP has subexponential time simulations unless EXPTIME has publishable proofs. Computational Complexity, 3(4):307-318, 1993.

[4] C. Damm. DET $=\mathrm{L}^{\# L}$. Technical Report Informatik-preprint 8, Fachbereich Informatik der Humboldt Universität zu Berlin, 1991.

[5] O. Goldreich. Foundation of Cryptography I: Basic Tools. Cambridge University Press, 2001.

[6] D. Grigoriev and A. Razborov. Exponential lower bounds for depth 3 arithmetic circuits in algebras of functions over finite fields. Applicable Algebra in Engineering, Communication and Computing, 10(6):467-487, 2000.

[7] R. Impagliazzo and A. Wigderson. $\mathrm{P}=\mathrm{BPP}$ if $\mathrm{E}$ requires exponential circuits: Derandomizing the XOR lemma. In Proceedings of Annual ACM Symposium on the Theory of Computing, pages 220-229, 1997.

[8] M. Jerrum and M. Snir. Some exact complexity results for straight-line computations over semirings. J. ACM, 29(3):874-897, 1982.

[9] Valentine Kabanets and Russell Impagliazzo. Derandomizing polyonmial identity tests means proving circuit lower bounds. In Proceedings of Annual ACM Symposium on the Theory of Computing, pages 355-364, 2003.

[10] H. Minc. Permanents. Addision-Wesley, 1978.

[11] K. Mulmulay and M. Sohoni. Geometric complexity theory, P vs. NP, and explicit obstructions. SIAM Journal on Computing, 31(2):496-526, 2002.

[12] D. Mumford. Algebraic Geometry I: Complex Projective Varieties. Springer-Verlag, 1976. Volume 221 of Grundlehren der Mathematischen Wissenschaften. 
[13] N. Nisan and A. Wigderson. Hardness vs. randomness. J. Comput. Sys. Sci., 49(2):149-167, 1994.

[14] N. Nisan and A. Wigderson. Lower bounds on arithmetic circuits via partial derivatives. Computational Complexity, 6(3):217-234, 1996/97.

[15] Ran Raz. Multi-linear formulas for permanent and determinant and of superpolynomial size. In Proceedings of Annual ACM Symposium on the Theory of Computing, pages 633-641, 2004.

[16] K. Regan. Understanding the Mulmuley-Sohoni approach to P vs. NP. Bulletin of the European Association for Theoretical Computer Science, 78:86-97, 2002. Lance Fortnow's Computational Complexity Column.

[17] J. T. Schwartz. Fast probabilistic algorithms for verification of polynomial identities. J. ACM, 27(4):701-717, 1980.

[18] J. Seiferas, M. Fischer, and A. Meyer. Separating nondeterministic time complexity classes. J. ACM, 25(1):146-167, 1978.

[19] A. Shpilka and A. Wigderson. Depth-3 arithmetic formulae over fields of charecteristic zero. In Proceedings of the Conference on Computational Complexity, pages 79-96, 1999.

[20] M. Sudan, L. Trevisan, and S. Vadhan. Pseudorandom generators without the XOR lemma. In Proceedings of Annual ACM Symposium on the Theory of Computing, pages 537-546, 1999.

[21] S. Toda. Counting problems computationally equivalent to the determinant. manuscript, 1991.

[22] S. Toda. PP is as hard as the polyonmial-time hierarchy. SIAM Journal on Computing, 20:865-877, 1991.

[23] L. Valiant, S. Skyum, S. Berkowitz, and C. Rackoff. Fast parallel computation of polynnomials using few processors. SIAM Journal on Computing, 12:641-644, 1983.

[24] L. G. Valiant. Completeness classes in algebra. In Proceedings of Annual ACM Symposium on the Theory of Computing, pages 249-261, 1979.

[25] V Vinay. Counting auxiliary pushdown automata and semi-unbounded arithmetic circuits. In Proceedings of the Structure in Complexity Theory Conference, pages 270-284. Springer LNCS 223, 1991.

[26] R. E. Zippel. Probabilistic algorithms for sparse polynomials. In EUROSCAM'r9, pages 216-226. Springer LNCS 72, 1979.

Department of Computer Science and Engineering Indian Institute of Technology, Kanpur 208016, India

E-mail: manindra@iitk.ac.in 